\title{
Psicooncología
}

ISSN: 1696-7240

\section{Evaluación de la percepción de autoeficacia en pacientes de oncología infantil}

\author{
Pilar Moreno Cañizares ${ }^{1}$; José Antonio Bueno Álvarez²
}

Recibido: 3 de mayo de 2019 / Aceptado el 10 de mayo de 2019

Resumen: Objetivo: Estudios realizados con pacientes adultos oncológicos revelan el efecto protector y terapéutico que la autoeficacia tiene en el afrontamiento de dicha enfermedad. No habiéndose estudiado la influencia de dicha variable en el cáncer infantil, se plantea este estudio con el objeto de evaluar la percepción de autoeficacia en estos pacientes. Método: Para ello se ha creado la Escala de Autoeficacia en Pacientes de Oncología Infantil (EAPOI) que arroja un índice de consistencia interna alfa de Cronbach de 0,889 . Resultados: Los resultados evidencian que la población objeto de estudio manifiesta una adecuada percepción de autoeficacia al situarse el $85 \%$ entre los niveles medio y muy alto; tan solo un $15 \%$ se sitúa en el nivel bajo. Conclusión: Estos resultados confirman la tendencia observada en los estudios de los últimos años que muestran una adecuada adaptación psicosocial del niño oncológico ante la enfermedad y sus tratamientos.

Palabras clave: autoeficacia, oncología, infancia.

\section{[en] Evaluation of perception of self-efficacy in pediatric oncology patients}

\begin{abstract}
Objective: Studies with adult oncology patients reveal the protective and therapeutic effect that self-efficacy has on facing the disease. The influence of this variable has not been studied in childhood cancer cases, thus this study is proposed in order to evaluate the perception of self-efficacy in these patients. Method: In doing so, Self-Efficacy Scale in Pediatric Oncology Patients has been created, providing an internal consistency index of Cronbach of .889. Results: The results of our investigation show that subjects believe themselves properly self-efficient to cope with their disease: $85 \%$ of them have medium-high level, and just $15 \%$ of them have low level. Conclusion: These results confirm the observed trend in recent studies that show an appropriate psychosocial adaptation of oncological children in the presence of the disease and its treatments.
\end{abstract}

Keywords: Self-efficacy, oncology, childhood.

Sumario. 1. Introducción 2. Método 2.1. Participantes 2.2. Procedimiento 2.3. Instrumento 3. Resultados 4. Discusión. 5. Limitaciones y líneas futuras de investigación. 6. Referencias bibliográficas

1 Pilar Moreno Cañizares. CPEE Hospital del Niño Jesús. Avda. Menéndez Pelayo 65, 28009 Madrid. E-mail: pilarmoka@gmail.com

2 José Antonio Bueno Álvarez. Departamento de Investigación y Psicología en Educación. Facultad de Ciencias de la Educación. Universidad Complutense de Madrid . Facultad de Educación.

E-mail:_alvarez@edu.ucm.es

* Dirección de correspondencia: Pilar Moreno Cañizares. CPEE Hospital del Niño Jesús. Avda. Menéndez Pelayo 65, 28009 Madrid. E-mail: pilarmoka@gmail.com 
Cómo citar: Moreno Cañizares P, Bueno Álvarez JA. Evaluación de la percepción de autoeficacia en pacientes de oncología infantil. Psicooncología 2019; 16: 387-403. doi:10.5209/psic.65598

\section{Introducción}

Según la Asociación Española Contra el Cáncer ${ }^{(1)}$, cada año en España 1100 niños reciben un diagnóstico de cáncer. El Registro Nacional de Tumores Infantiles ${ }^{(2)}$ publica, en sus últimas estadísticas de los años 1980 a 2017, que los tipos de tumores que se suelen dar en la infancia en mayor medida entre 0-14 años son: leucemias: $27 \%$ (siendo la más frecuente la leucemia linfoblástica aguda), tumores del sistema nervioso central: $21 \%$ y linfomas: $13 \%$.

A lo largo de su enfermedad y tratamiento el niño con cáncer se enfrenta a procedimientos médicos dolorosos, a frecuentes hospitalizaciones, a importantes efectos secundarios derivados de los tratamientos (algunos reversibles como el dolor, los vómitos o la caída de cabello pero otros permanentes como la amputación de alguno de los miembros o el daño cerebral). En definitiva, el niño se enfrenta a una ruptura real de su vida hasta el momento del diagnóstico

Desde que en 1977 Albert Bandura introdujera el concepto de autoeficacia, muchas han sido las investigaciones que lo han analizado y desarrollado desde diferentes campos ${ }^{(3)}$. A través de dichos estudios se considera la autoeficacia como mediador cognitivo esencial que dirige la conducta humana. La autoeficacia es, por tanto, una creencia sobre la propia competencia o efectividad personal y está ligada a cada área concreta del funcionamiento humano.

Su repercusión en el ámbito de la salud ha sido ampliamente documentada a través de numerosos estudios en los que se revelan los beneficios que una alta autoeficacia tiene para el funcionamiento humano de las personas y su bienestar ${ }^{(3)}$. Concretamente en el caso del cáncer en adultos, diferentes investigaciones analizadas muestran, entre otros aspectos, la influencia positiva de la autoeficacia en: el afrontamiento de secuelas corporales ${ }^{(4,5)}$; la adherencia a los tratamientos médicos $^{(6)}$; o en el ajuste psicológico y bienestar físico de los pacientes ${ }^{(7,8)}$. Así también, como que una adecuada información médica sobre la enfermedad o el tratamiento o la posibilidad de expresar dudas o temores sobre ambos aspectos, incrementa la autoeficacia de los pacientes en su afrontamiento de la enfermedad ${ }^{(9)}$ . Sin embargo, encontramos un vacío en estas investigaciones: no se ha estudiado la influencia de dicha variable en el cáncer infantil. Ante el vacío de información sobre este tema y, constatados los beneficios que una alta autoeficacia parece proporcionar en el afrontamiento del cáncer en adultos, nos preguntamos si no parece razonable inferir que dichos beneficios puedan darse también en el caso de los pacientes pediátricos.

En este sentido el objetivo de la investigación que presentamos en este artículo fue construir y validar un instrumento de medida que proporcionara un diagnóstico fiable y válido de este constructo para, posteriormente, analizar las respuestas de los sujetos al mismo con el objeto de poder evaluar la percepción de autoeficacia de estos pacientes. 


\section{Método}

\subsection{Participantes}

La muestra que participó en este estudio estaba compuesta por 110 sujetos, de los cuales el $59 \%$ eran niños y el $41 \%$ niñas. Las edades de los participantes estaban comprendidas entre los 8 y los 14 años, con una media de 11.5 y una desviación típica de 1.9.

Respecto de la procedencia de los sujetos, éstos estaban ingresados en los Servicios de Oncología Pediátrica (en planta o en hospital de día) de los siguientes hospitales públicos españoles: 41\% Hospital Infantil Universitario Niño Jesús de Madrid; 45\% Hospital Universitario La Paz de Madrid; 6\% Hospital Universitari i Politècnic La Fe de Valencia; 8\% Hospital Universitario Cruces de Bilbao. La gran mayoría de los participantes $(80 \%)$ se encontraba en fase de tratamiento y el $20 \%$ restante, en fase de recaída (se había vuelto a desarrollar la enfermedad).

Tabla 1. Características demográficas y clínicas de la muestra

\begin{tabular}{llll}
\hline \multicolumn{1}{c}{ Variable } & \multicolumn{1}{c}{ Categoría } & $\mathrm{N}$ & $\%$ \\
\hline Edad & $8-11$ & 55 & 50 \\
& $12-14$ & 55 & 50 \\
Sexo & Hombre & 65 & 59 \\
& Mujer & 45 & 41 \\
Fase & Tratamiento & 88 & 80 \\
& Recaída & 22 & 20 \\
Total & & 110 & \\
\hline
\end{tabular}

\subsection{Procedimiento}

Para poder acceder a la muestra, fue necesaria la aprobación del protocolo de investigación del estudio por parte de los distintos Comités Éticos de Investigación Clínica de cada hospital, así como por los Jefes de Servicio de las distintas unidades de Oncología Pediátrica de los citados hospitales.

La participación fue voluntaria, previo consentimiento de los padres de los sujetos participantes, manteniéndose una estricta confidencialidad en el tratamiento y uso de los datos obtenidos. El proceso de recogida de los mismos se llevó a cabo de manera individualizada. Se leyeron las instrucciones a cada participante y se aclararon sus dudas. Se insistió en la confidencialidad y en la sinceridad de las respuestas, con el fin de maximizar la validez de la información obtenida. Las instrucciones dadas a los sujetos participantes consistieron en que rellenaran, en primer lugar, las casillas referidas a edad y sexo. Posteriormente, completaban los diferentes ítems de la EAPOI indicándoles que, en una escala de respuesta con numeración de 1 a 10, rodearan el valor que más se ajustara a cuánto se creían capaces en ese momento de realizar las cosas descritas en cada ítem. 
Todas las escalas fueron aplicadas, en cada hospital, por una persona voluntaria previamente entrenada para ello. Dicha persona, además, recogía los datos proporcionados por los padres, referidos a fecha de diagnóstico y fase de la enfermedad en la que el sujeto se encontraba.

\subsection{Instrumento}

El instrumento que hemos construido se denomina Escala de Autoeficacia en Pacientes de Oncología Infantil (EAPOI) y evalúa la percepción de autoeficacia de estos pacientes en el afrontamiento de situaciones o comportamientos de especial dificultad derivados de su enfermedad y/o tratamiento.

El proceso de construcción de dicha escala se desarrolló en dos etapas ${ }^{(3)}$. En la primera, tras la observación y conversación con diferentes pacientes de oncología infantil, clarificamos cuáles eran las conductas más difíciles de afrontar por estos pacientes y, una vez concretadas, se fueron integrando en la redacción de los diferentes ítems de la escala. Este proceso dio lugar a un listado inicial de 30 ítems, formulados de manera que en cada uno de ellos el sujeto juzga su capacidad en ese momento de realizar la conducta especificada.

Posteriormente se contactó con diferentes expertos en el tema (médicos, enfermeras, maestros de aula hospitalaria), que de forma individual revisaron y valoraron los 30 ítems citados. La evaluación se centró en valorar la relevancia y claridad de cada ítem. Así también, se les pidió su opinión sobre la modificación, eliminación o inclusión de algún ítem nuevo. Una vez consideradas todas las valoraciones de los expertos, la escala resultante acabó integrada por 22 ítems.

Estos ítems se presentan en la EAPOI en orden aleatorio, con una escala de respuesta que va de 1 a 10, siendo: 1 "Nada capaz" y 10 "Totalmente capaz", tipo de escala recomendado por Bandura ${ }^{(10)}$ pues de esta forma los sujetos estiman la fortaleza de sus creencias de eficacia para desempeñar las conductas señaladas.

En la segunda etapa se analizaron las propiedades psicométricas de la EAPOI con el objeto de comprobar la validez y fiabilidad de la misma.

\section{Resultados}

Una vez aplicada la escala a la muestra participante y recogida toda la información se llevaron a cabo dos tipos de análisis de $\operatorname{los}_{\text {datos }}{ }^{(3)}$. En primer lugar, para la selección de los ítems definitivos, se utilizaron dos métodos complementarios ${ }^{(11-13)}$ : (1) análisis de ítems mediante el procedimiento de la correlación ítem-total y cálculo de la fiabilidad utilizando el coeficiente alfa de Cronbach; (2) análisis factorial exploratorio mediante el método de Componentes Principales aplicando después una Rotación ortogonal Varimax.

Antes de proceder a estos análisis, consideramos un aspecto básico como es la normalidad de la distribución. Al superar nuestra muestra las 50 unidades, recurrimos al estadístico D Kolmogorov-Smirnov con una corrección de la significatividad de la normalidad de Lilliefors. Al aplicar esta prueba obtenemos los resultados que se muestran en la tabla 2 . 
Tabla 2. Pruebas de normalidad

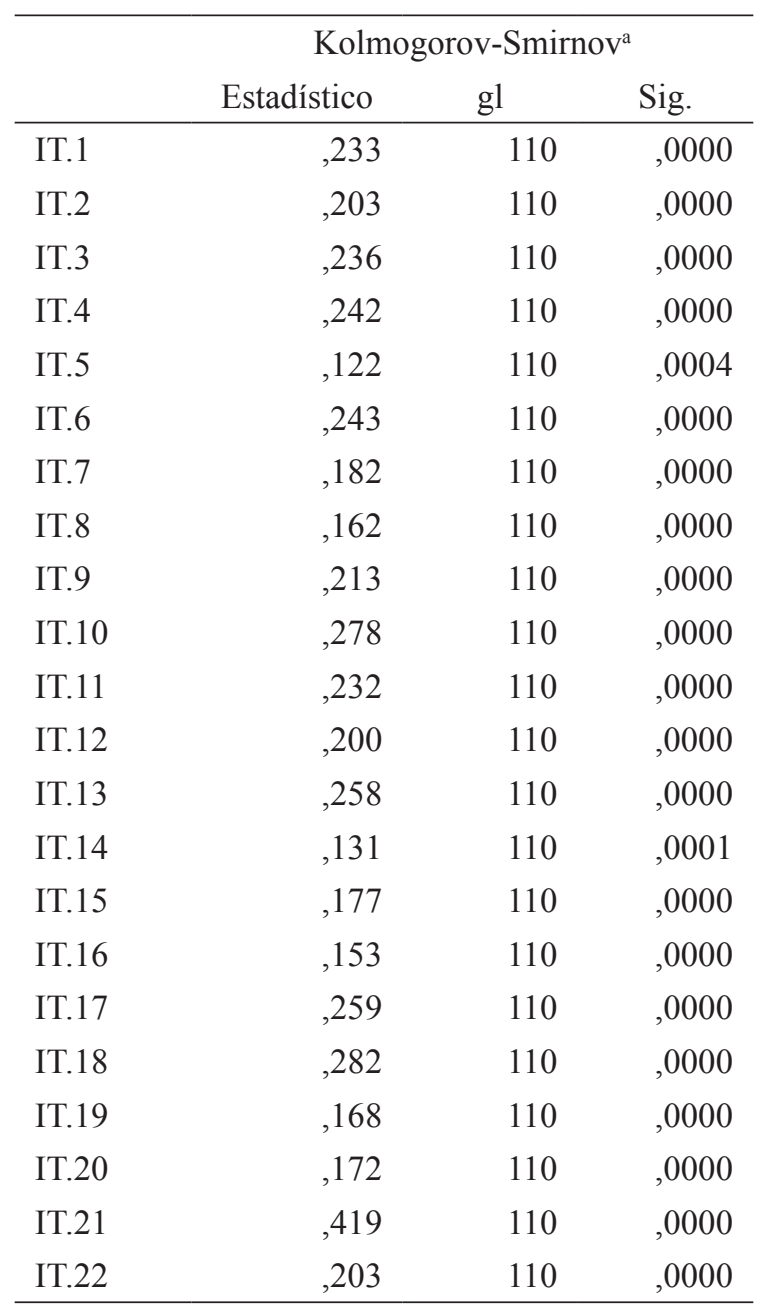

a. Corrección de la significación de Lilliefors

Como se observa, los niveles de significación de Lilliefors son en todos los ítems 0,000 . Esto supone el rechazo de la hipótesis nula de normalidad en todos los ítems considerados para el análisis.

Con respecto a la fiabilidad de la escala, el índice de consistencia interna alfa de Cronbach de la EAPOI es de 0,889. La Tabla 3 muestra la correlación ítem-total corregida así como la fiabilidad de la escala si suprimimos el ítem.

Siguiendo las recomendaciones de Nunnally y Bernstein ${ }^{(14)}$, es necesario un valor de 0,30 como mínimo para la selección de los ítems. Al observar la tabla 2,10 ítems 7,11 y 12 presentan los valores más bajos $(0,304,0,337$ y 0,362 respectivamente) pero por encima de 0,30 . A continuación, vamos a ir suprimiendo estos ítems para comprobar si se produce un incremento de la fiabilidad de la escala (tabla 4). 
Tabla 3. Correlación elemento-total corregida y Alfa de Cronbach si se elimina el elemento

\begin{tabular}{ccc}
\hline TE CREES CAPAZ DE & $\begin{array}{c}\text { Correlación } \\
\text { elemento-total } \\
\text { corregida }\end{array}$ & $\begin{array}{c}\text { Alfa de Cronbach } \\
\text { si se elimina el } \\
\text { elemento }\end{array}$ \\
\hline
\end{tabular}

IT.1: tomarte "sin protestar" las medicinas que te , 479 , 885 manda el médico?

IT.2: continuar con tus estudios ahora que tienes esta enfermedad?

IT.3: contarle a tus padres cómo te sientes: triste, enfadado, cansado, etc.)?

IT.4: preguntar a los médicos o enfermeras cosas que quieras saber sobre tu enfermedad (cómo se llama, en qué consiste, etc.)?

IT.5: concentrarte ahora en tus tareas escolares?

IT.6: estar tranquilo mientras te hacen una cura o un análisis de sangre?

IT.7: poder permanecer unos días en tu habitación del hospital sin salir (como aislado)?

IT.8: esforzarte en tus estudios de la misma manera que antes de enfermar?

IT.9: contarle a tus padres si algo te preocupa o asusta de tu enfermedad?

IT.10: hacer todo lo que el médico te aconseje para ponerte bueno?

IT.11: salir a jugar con tus amigos?

IT.12: que te vean tus amigos o compañeros de clase aunque tu aspecto físico haya cambiado (por ejemplo: la caída del pelo, la hinchazón, etc.)? 


\begin{tabular}{ccc}
\hline ¿TE CREES CAPAZ DE & $\begin{array}{c}\text { Correlación } \\
\text { elemento-total } \\
\text { corregida }\end{array}$ & $\begin{array}{c}\text { Alfa de Cronbach } \\
\text { si se elimina el } \\
\text { elemento }\end{array}$ \\
\hline
\end{tabular}

IT.13: hacer preguntas a los médicos o enfermeras , 609 , 882 sobre el tratamiento que te van a dar para ponerte bien (en qué va a consistir, cómo te vas a encontrar con él, etc.)?

IT.14: realizar las mismas tareas en casa que antes de enfermar (hacer la cama, poner la mesa, etc.)?

IT.15: hablar de algo que te preocupe o asuste de tu enfermedad con personas que no sean tus padres (hermanos, primos, amigos, profesores, médicos, etc.)?

IT.16: aceptar o sobrellevar lo que no te deja hacer , 610 , 881 el tratamiento o la enfermedad (por ejemplo jugar con tus amigos, ir al colegio, etc.?

IT.17: continuar con tus entretenimientos o juegos de siempre?

IT.18: permanecer tranquilo durante el tratamiento de quimioterapia/radioterapia?

IT.19: recordar bien la información que te explica el profesor o que lees en los libros?

IT.20: enfrentarte a los cambios físicos o molestias provocados por tu enfermedad o su tratamiento (por ejemplo la caída de pelo, la hinchazón, dolor...)?

IT.21: disfrutar y reírte con las cosas que te gustan? jugar, pasear,...) y no pasarte el tiempo tumbado? 
Tabla 4. Correlación elemento-total corregida y Alfa de Cronbach sin ítem 7, 11 y 12

\begin{tabular}{lrr}
\hline & $\begin{array}{c}\text { Correlación elemento-total } \\
\text { corregida }\end{array}$ & \multicolumn{2}{c}{$\begin{array}{c}\text { Alfa de Cronbach si se } \\
\text { elimina el elemento }\end{array}$} \\
\hline IT.1 &, 519 &, 889 \\
IT.2 &, 477 &, 890 \\
IT.3 &, 497 &, 889 \\
IT.4 &, 558 &, 888 \\
IT.5 &, 473 &, 890 \\
IT.6 &, 450 &, 891 \\
IT.8 &, 520 &, 889 \\
IT.9 &, 591 &, 887 \\
IT.10 &, 560 &, 888 \\
IT.13 &, 625 &, 886 \\
IT.14 &, 476 &, 891 \\
IT.15 &, 595 &, 887 \\
IT.16 &, 558 &, 887 \\
IT.17 &, 557 &, 888 \\
IT.18 &, 527 &, 889 \\
IT.19 &, 485 &, 890 \\
IT.20 &, 456 &, 891 \\
IT.21 &, 537 &, 890 \\
IT.22 &, 676 &, 884 \\
\hline
\end{tabular}

A través del análisis de ítems concluimos que la supresión de los ítems con valores más bajos: 7, 11 y 12 aumentaría el índice de fiabilidad, aunque de forma muy escasa, de 0,889 a 0,894 .

Antes de proceder al análisis factorial comprobamos la pertinencia del mismo a través del índice KMO o medida de adecuación muestral de Kaiser-Meyer-Olkin. El valor obtenido de 0,821 se considera adecuado. No tuvimos en cuenta la prueba de esfericidad de Bartlett pues ésta exige que los datos provengan de una distribución normal.

Tras el análisis factorial, los factores sin rotar muestran que todos los ítems presentan pesos superiores a 0,30 en el primer factor general; sin embargo, seis de ellos $\left(7,11,12,5,2\right.$ y 8) los tienen mayores en otros factores ${ }^{(13)}$.

Con ambos criterios: correlación ítem-total y correlación ítem primer factor general (Componentes Principales) los resultados indican que estadísticamente se podrían suprimir los ítems 7, 11, 12, 5, 2 y 8; no obstante, conceptualmente esto supondría una pérdida importante de información pues aspectos como el aislamiento, el afrontamiento de cambios en la imagen corporal o el funcionamiento personal, que consideramos fundamentales en el tema que estamos tratando, no quedarían bien representados en la escala. 
Tras la rotación de los factores (tabla 5), la solución factorial resultante no nos resulta satisfactoria ni en términos estadísticos ni conceptuales. A continuación analizamos el porqué.

Tabla 5. Matriz de componentes rotados ${ }^{\mathrm{a}}$

\begin{tabular}{|c|c|c|c|c|c|c|}
\hline & \multicolumn{6}{|c|}{ Componente } \\
\hline & 1 & 2 & 3 & 4 & 5 & 6 \\
\hline IT.4 & ,799 & ,089 &, 151 &, 160 &, 047 &, 004 \\
\hline IT.13 & ,788 & , 150 & , 180 & ,281 &,- 039 &,- 071 \\
\hline IT.15 & ,719 & ,043 & , 184 & , 186 &, 260 &, 142 \\
\hline IT.9 & ,609 & , 145 & ,118 & ,008 &, 532 & , 154 \\
\hline IT.3 &, 580 & ,115 &, 044 & ,087 & ,360 &,- 098 \\
\hline IT.5 & ,027 & ,894 &,- 023 & ,076 & , 185 &,- 013 \\
\hline IT. 8 &, 250 & ,830 & ,046 & ,081 &,- 008 &, 062 \\
\hline IT. 2 &,- 022 & ,770 &,- 031 & ,267 & ,193 & , 124 \\
\hline IT.19 &, 167 & ,691 & ,258 &,- 083 & , 196 &,- 118 \\
\hline IT.12 & 035 & ,007 & ,853 &,- 023 & , 106 &, 016 \\
\hline IT.20 & ,305 & ,067 & ,743 & ,011 & , 185 & ,299 \\
\hline IT.11 & , 128 & ,023 & ,629 & ,353 &,- 309 &,- 163 \\
\hline IT.16 & ,304 & , 197 &, 558 & ,286 & ,089 &, 146 \\
\hline IT.21 & ,134 & ,028 &, 524 & ,513 & ,234 &,- 035 \\
\hline IT.18 & , 164 &, 113 &, 076 & ,717 & ,219 & ,203 \\
\hline IT.6 & , 177 &,- 054 &, 064 & ,675 & ,281 & ,093 \\
\hline IT.14 &, 225 & ,363 & ,114 & ,632 &,- 183 &,- 077 \\
\hline IT.10 & ,271 & , 190 & ,095 & , 149 & ,693 & ,123 \\
\hline IT.1 & , 109 & ,232 &, 057 & ,269 & ,676 &,- 083 \\
\hline IT.22 & ,284 & ,270 & ,247 & ,421 &, 428 &,- 255 \\
\hline IT.7 & ,092 & , 103 & ,233 & ,206 &, 081 &, 852 \\
\hline IT.17 & ,221 & ,202 & ,282 &, 445 & ,296 &,- 486 \\
\hline Varianza & $14,2 \%$ & $13,8 \%$ & $12,1 \%$ & $11,8 \%$ & $9,9 \%$ & $6,1 \%$ \\
\hline
\end{tabular}

Método de extracción: Análisis de componentes principales.

Método de rotación: Normalización Varimax con Kaiser.

a. La rotación ha convergido en 8 iteraciones.

Siguiendo las recomendaciones teóricas, Nunnally y Bernstein ${ }^{(14)}$ señalan que un factor está fuertemente definido cuando al menos cuatro de las variables que se correlacionan altamente con ese factor no se correlacionan más de 0,30 con cualquier otro factor y que incluso las variables que le definen deben correlacionarse con él por encima de 0,50. Además, añaden que si un factor no es tan fuerte es mejor ignorarlo porque las soluciones factoriales que carecen de tales agrupamientos definidos suelen ser deficientes al ser altamente dependientes del método que se utilice. Costello y 
Osborne ${ }^{(15)}$ indican que si hay varios ítems con cargas fuertes (a partir de 0,50) en varios factores una buena opción podría ser eliminarlos. También añaden que un factor con menos de tres ítems es generalmente débil e inestable; por el contrario, factores con cinco o más ítems con cargas fuertes (.50 o mayor) son indicadores de un factor sólido.

Tal como muestra la tabla 5, de los seis componentes de nuestra escala solamente uno, concretamente el segundo, cumple los requisitos citados. El resto de componentes incurren en una o varias de las siguientes incidencias:

- Alguno de los ítems satura más de 0,30 en otro factor: componentes 1 (ítems 9 y 3 ), 3 (ítems 20, 11, 16 y 21), 4 (ítem14), 5 (ítem 22) y 6 (ítem 17). Incluso alguno de estos ítems satura más de 0,50 en otro factor: componentes 1 (ítem 9) y 3 (ítem 21).

- Alguno de los ítems no se correlaciona con el factor al que pertenece por encima de 0,50: componentes 5 (ítem 22) y 6 (ítem 17).

- Uno de los factores está formado únicamente por dos ítems: componente 6.

- Dificultades en el análisis conceptual de los componentes 4 y 6: en el componente 4, el ítem 14 no guarda relación conceptual con los otros ítems que configuran el factor $\mathrm{y}$, en el componente 6 , la relación conceptual de ambos ítems hay que forzarla.

Siendo rigurosos con las recomendaciones teóricas citadas, comprobamos que únicamente el componente 2 es lo suficientemente fuerte estadísticamente hablando para ser tenido en cuenta $y$, a nivel conceptual, ya hemos mencionado las deficiencias que presentan los componentes 4 y 6 .

Valorando todos los aspectos estadísticos y conceptuales que hemos ido analizando consideramos que, por un lado, al ser suficientemente elevado el índice de consistencia interna de la escala (alfa de Cronbach: 0,889) y sopesando la pérdida de información que supondría la eliminación de las citadas variables, se decide mantener los 22 ítems iniciales. Por otro lado, no juzgamos pertinente la consideración diferenciada de los componentes de la escala tanto por las deficiencias expuestas como por no haber sido contemplados en nuestra concepción inicial del instrumento al considerar como un todo a los aspectos de especial dificultad a los que se enfrenta el niño oncológico (ámbito médico, emocional, educativo, social...). Además estimamos adecuado tanto el número de ítems como el tiempo de ejecución (5 o 10 minutos).

En segundo lugar, para poder describir y comparar las creencias de autoeficacia de los participantes, se realizó, por un lado, un análisis de los estadísticos descriptivos de cada ítem y, por otro lado, se llevó a cabo un análisis de contraste de hipótesis para ver si las respuestas de los sujetos diferían en función de las variables recogidas en la EAPOI: sexo, edad y fase de la enfermedad. Para ello se utilizaron las pruebas U de Mann-Whitney y H de Kruskal-Wallis.

Para poder clasificar las puntuaciones de los sujetos en la EAPOI se eligió el criterio estadístico restrictivo que suele ser utilizado en el ámbito de la investigación ${ }^{(16)}$. Se basa en los percentiles 15, 80 y 95 que corresponden a un nivel bajo, medio, alto o muy alto de autoeficacia respectivamente. En este caso dichos percentiles se corresponden con las puntuaciones $\mathrm{PC}_{15}=150, \mathrm{PC}_{80}=198$ y $\mathrm{PC}_{95}=211$ de la EAPOI. Por tanto, un nivel bajo de autoeficacia se corresponde con puntuaciones entre 22 y 
149 , un nivel medio se corresponde con puntuaciones entre 150 y 197 , un nivel alto se corresponde con puntuaciones entre 198 y 210 y, un nivel muy alto de autoeficacia se corresponde con puntuaciones entre 211 y 220.

Como se observa en la tabla 6 , los resultados obtenidos por los 110 sujetos que componen la muestra fluctuaron entre un nivel bajo de autoeficacia (puntuación 98) y un nivel muy alto (puntuación 219). Por categorías, la puntuación más baja la encontramos en el grupo de 8-11 años, en el grupo de mujeres y en el grupo de tratamiento. La media general se sitúa en un nivel medio de autoeficacia (174.95). La puntuación media obtenida por grupo de edad, por sexo y por fase fue muy similar a la observada en la media general, no encontrándose diferencias estadísticamente significativas en ninguna de estas variables.

Tabla 6. Estadísticos descriptivos de la puntuación total, por grupo de edad, sexo y fase de la enfermedad

\begin{tabular}{lllcccc}
\hline Variable & Categoría & $\mathrm{N}$ & Media & Desv. Típica & Mínimo & Máximo \\
\hline Edad & $8-11$ & 55 & 173,78 & 33,15 & 98 & 219 \\
& $12-14$ & 55 & 176,11 & 17,85 & 125 & 208 \\
\multirow{2}{*}{ Sexo } & Hombre & 65 & 178,51 & 24,71 & 100 & 219 \\
& Mujer & 45 & 169,80 & 28,44 & 98 & 215 \\
\multirow{2}{*}{ Fase } & Tratamiento & 88 & 174,18 & 26,48 & 98 & 215 \\
& Recaída & 22 & 178,00 & 27,13 & 111 & 219 \\
Total & & 110 & 174,95 & 26,53 & 98 & 219 \\
\hline
\end{tabular}

\section{Discusión}

El conjunto de los 22 ítems que conforman la EAPOI incluyen siete grandes aspectos o temas. A continuación se analiza la importancia de la inclusión de los mismos en la escala así como los resultados obtenidos en el estudio.

Búsqueda y comprensión de información médica sobre la enfermedad que padecen así como de los tratamientos que van a recibir.

A través de diferentes estudios analizados se avala la importancia de que el niño posea una información clara, correcta y adecuada a su nivel de desarrollo, tanto de la enfermedad que padece como del tratamiento que va a recibir ${ }^{(17-22)}$. Conocer qué le pasa y qué le van a hacer, permite al paciente comprender y afrontar mejor la situación que está atravesando, además, este conocimiento le va a llevar a implicarse más activamente en el cuidado de su salud ${ }^{(21-23)}$. 
Por el contrario, como señala $\mathrm{Die}^{(24)}$ proporcionar información contradictoria o imprecisa puede generar tensión o angustia en el menor y, como explica Domínguez ${ }^{(19)}$, da lugar a que los niños elaboren falsas creencias como por ejemplo que el cáncer se contagia o que es el resultado de haber salido sin abrigo a la calle... lo que le llevaría a pensar que es el responsable de su enfermedad.

Este aspecto está recogido en los ítems 4 y 13 de la EAPOI y en ellos los sujetos de nuestra muestra obtuvieron un nivel medio de autoeficacia $(\bar{X}=8,6$. Nótese que aquí la media no se refiere al total de la escala sino a la media en estos ítems. Un nivel medio de autoeficacia se corresponde con puntuaciones entre 6,9 y 8,9 ).

Capacidad del sujeto de enfrentarse a los procedimientos médicos requeridos por el tratamiento.

Durante el protocolo del diagnóstico y el tratamiento posterior el paciente de oncología infantil es sometido a diferentes procedimientos médicos. Nosotros nos centramos en los más comunes a las distintas patologías y los menos invasivos: análisis de sangre, curas, quimioterapia y radioterapia. Aunque los citados procedimientos son menos invasivos no dejan de poder ser fuente generadora de estrés para estos pacientes, fundamentalmente por los síntomas asociados que estos pueden provocar: dolor, vómitos, mareos, fatiga...

Investigaciones realizadas sobre los aspectos más negativos de los tratamientos ${ }^{(25,26)}$ señalan que en niños hasta 12 años el síntoma más estresante es el dolor y en los mayores de 13 años, las náuseas ${ }^{(25)}$.

La dificultad que puede llevar a un paciente de estas edades a enfrentarse a dichos procedimientos médicos es la que nos llevó a incluir este contenido en la EAPOI a través de los ítems 6 y 18. En ellos los sujetos muestran un nivel medio de autoeficacia $(\bar{X}=8,4)$. Es decir, los sujetos se perciben adecuadamente autoeficaces en su capacidad de permanecer tranquilos ante una cura, un análisis de sangre o el tratamiento de quimioterapia/radioterapia.

Además, el análisis de contraste de hipótesis realizado señala en el ítem 18 la existencia de diferencias estadísticamente significativas entre los sujetos en función de la fase de la enfermedad que se encuentran $(p=0,001 ; a=0,05)$. Así, los sujetos en fase de recaída $(\bar{X}=9,7)$ se perciben más autoeficaces que los sujetos en fase de tratamiento $(\bar{X}=8,2)$ al enfrentarse a los tratamientos de quimioterapia y/o radioterapia. Esta diferencia podría explicarse por la experiencia y conocimientos previos que sobre este tipo de tratamientos tiene el paciente en fase de recaída que le permitirían enfrentarse a ellos con una mayor seguridad y confianza.

\section{Adherencia a los tratamientos médicos.}

Si para cualquier enfermedad es fundamental el cumplimiento de las prescripciones médicas, todavía lo es más para una enfermedad de la gravedad del cáncer. Además, el aumento en las tasas de supervivencia del enfermo oncológico se debe principalmente al seguimiento del mismo.

Sin embargo, para los niños de estas edades los continuos ingresos, los tratamientos intrusivos y dolorosos, los importantes efectos secundarios derivados 
de los mismos, las constantes revisiones y un gran número de cuidados personales, dificultan la conducta de adherencia en el niño, manifestando en ocasiones comportamientos negativos como quejas, llantos, oposición, resistencia y poca colaboración $^{(21)}$.

En dicho aspecto, recogido en los ítems 1,10 y 22, los sujetos muestran un nivel medio de autoeficacia $(\bar{X}=8,4)$, es decir, los sujetos se perciben adecuadamente autoeficaces en su capacidad de cumplimiento de aquellos comportamientos prescritos por su médico.

Además, el análisis de contraste de hipótesis realizado señala en el ítem 1 la existencia de diferencias estadísticamente significativas entre los sujetos en función de la edad $(p=0,035 ; a=0,05)$. Así, los sujetos de $12-14$ años $(\bar{X}=8,5)$ se perciben más autoeficaces que los sujetos de 8-11 años $(\bar{X}=7,5)$ en adherencia a la toma de su medicación. Esta diferencia por edad puede ser explicada desde el punto de vista evolutivo por un mayor autocontrol, en los sujetos de edades superiores, de los propios deseos en aras de un bien mayor: recuperar la salud.

\section{Afrontamiento de los cambios fisicos originados por la enfermedad/tratamiento.}

Este aspecto fue incluido por la dificultad que puede entrañar para algunos niños, por un lado, afrontar/aceptar estos cambios corporales y, por otro lado, tolerar el ser visto por otras personas tras sufrir estos cambios. Esta dificultad, como se señala desde diferentes investigaciones ${ }^{(20,21,25,27))}$ podría desembocar en problemas de autoestima, autoconfianza, aislamiento social o estrés emocional.

En este aspecto los sujetos muestran un nivel medio de autoeficacia $(\bar{X}=7,5)$, es decir, los sujetos se perciben moderadamente autoeficaces en su capacidad de afrontar los cambios físicos experimentados durante la enfermedad (ítem 20) así como en su capacidad de tolerar el ser visto por los demás a pesar de estos cambios (ítem 12).

Además, el análisis de contraste de hipótesis realizado señala la existencia de diferencias estadísticamente significativas entre los sujetos en función del sexo (ítem 20: $p=0,019 /$ ítem 12: $p=0,003 ; a=0,05$ ). Así, las mujeres (ítem 20: $\bar{X}=7 /$ ítem 12: $\bar{X}=6,4)$ se perciben menos autoeficaces que los hombres (item $20: \bar{X}=8,2 /$ item 12 : $\bar{X}=7,9)$ en ambos aspectos. Este resultado está en consonancia con los hallazgos encontrados en otros estudios en los que se señala que las niñas parecen presentar más dificultades para afrontar los cambios en su imagen corporal ${ }^{(27)}$. Esto podría explicarse por la influencia de variables culturales que llevan a las mujeres a una mayor preocupación por su apariencia física ${ }^{(28)}$.

Aceptación de las restricciones que conlleva el proceso de tratamiento.

Dichas restricciones normalmente se dirigen a preservar la delicada salud de estos pacientes y se refieren a la no asistencia al centro en el que están escolarizados, a las limitaciones en contactos con familiares o amigos y a los períodos de aislamiento en su habitación del hospital.

Estas restricciones suponen un cambio en el funcionamiento social de los sujetos $y$, como se alerta en diversas investigaciones, podrían originar dificultades en sus 
interacciones así como problemas de ansiedad(21,29); por su parte, el estudio de Hedström et al. ${ }^{(25)}$ también señala el confinamiento como uno de los factores más estresantes para los pacientes infantiles.

En esta variable (ítems 7 y 16), los sujetos muestran un nivel medio de autoeficacia $(\bar{X}=7,3)$, es decir, los sujetos se perciben moderadamente autoeficaces en su capacidad de aceptar o sobrellevar las restricciones que conlleva la enfermedad.

\section{Búsqueda de apoyo emocional.}

Que el niño sea capaz comunicar cómo se siente, qué le preocupa, qué le da miedo... va a permitir que se le proporcione la ayuda necesaria en cada momento.

En este aspecto (ítems 3, 9 y 15) los sujetos muestran un nivel medio de autoeficacia $(\bar{X}=7,9)$ disminuyendo este nivel cuando la comunicación va dirigida hacia personas distintas a los padres. Diferentes estudios consideran el apoyo parental como factor de protección que ayuda al niño a soportar los retos que plantea la enfermedad y les hace emocionalmente más fuertes ${ }^{(30,31)}$. Este apoyo incondicional de los padres, observado por los niños, puede ser la clave que les permita abrirse hacia ellos y expresarles sus sentimientos y preocupaciones.

Además, el análisis de contraste de hipótesis realizado señala en el ítem 3 la existencia de diferencias estadísticamente significativas entre los sujetos en función del sexo $(p=0,032 ; a=0,05)$. Así, las mujeres $(\bar{X}=7,8)$ se perciben menos autoeficaces que los hombres $(\bar{X}=8,7)$ en su capacidad de comunicar sus sentimientos a sus padres. Este hallazgo podría explicarse como un factor de protección de las niñas hacia sus padres. Uno de los resultados de la investigación sobre resiliencia en madres e hijos con cáncer de González-Arratia et al. ${ }^{(30)}$ muestra ligeras diferencias a favor de las niñas como más empáticas; así éstas, al observar la angustia de sus padres, intentarían protegerles no revelando su propio grado de angustia ${ }^{(32,33)}$.

\section{Actividades o rutinas propias de la infancia.}

Aquí se incluyen: la educación, los juegos y las pequeñas tareas domésticas encomendadas en el hogar.

Estas actividades se consideran esenciales para la salud física y psicológica de cualquier niño y, además, para estos pacientes constituyen normalizadores de su vida pues son elementos que aportan al sujeto la sensación de continuar con su vida de siempre.

En este aspecto (ítems 2, 5, 8, 11, 14, 17, 19 y 21) los sujetos muestran un nivel medio de autoeficacia $(\bar{X}=7,7)$, percibiéndose adecuadamente autoeficaces en la realización de estas actividades propias de la infancia. En este punto encontramos el ítem de menor puntuación de la escala, que se refiere a la realización de las pequeñas tareas domésticas $(\bar{X}=6,9)$ y, el de mayor puntuación $(\bar{X}=9,4)$ en el que los sujetos muestran un nivel alto de autoeficacia en su percepción de poder seguir disfrutando de la vida a pesar de la enfermedad.

A modo de conclusión podemos señalar que los resultados de nuestra investigación evidencian que los sujetos de nuestra muestra se perciben adecuadamente autoeficaces para afrontar aquellos aspectos de especial dificultad derivados de su enfermedad o tratamiento. Así el 85\% de los sujetos se sitúan entre los niveles medio y muy alto; tan 
solo un $15 \%$ se sitúa en el nivel bajo. De estos resultados podemos inferir que están en consonancia con los estudios realizados en los últimos años, en los que se indica que el niño oncológico muestra una adecuada adaptación psicosocial ${ }^{(17,20,27,30,31,33-39)}$.

\section{Limitaciones y líneas futuras de investigación}

Una vez finalizada la investigación y habiendo reflexionado sobre el proceso realizado y los resultados obtenidos, resaltamos aquí dos de las limitaciones encontradas por su relevancia ${ }^{(3)}$ :

En primer lugar, aunque no se pudo tener en cuenta la variable (incluida en la escala): tiempo transcurrido desde el diagnóstico porque en algunos hospitales no se anotó, consideramos importante registrarla pues es una variable que permitiría conocer si el nivel de autoeficacia de los sujetos varía a lo largo del tiempo y, si varía, cómo. De esta forma podremos conocer en qué momento son más vulnerables los pacientes.

En segundo lugar, consideramos que en posteriores análisis deberían incluirse variables no contempladas en la EAPOI como diagnóstico (tipo de cáncer) y tipo de tratamiento, pues la revisión de artículos sobre niños afectos de tumores cerebrales y leucemias $\mathrm{u}$ otros tumores que han recibido quimioterapia intratecal y /o irradiación cráneo espinal, les señalan como grupo de riesgo que requiere un seguimiento más específico.

De cara a futuras investigaciones, consideramos que el siguiente paso a realizar siguiendo esta línea de investigación es estudiar qué papel juega la percepción de autoeficacia del paciente oncológico infantil en el afrontamiento de su enfermedad y tratamiento. Planteamos la hipótesis de que las creencias de autoeficacia constituyen uno de los mecanismos que ayudan a estos pacientes a hacer frente a su enfermedad.

\section{Referencias bibliográficas}

1. Asociación Española Contra el Cáncer. Información sobre el cáncer. [Internet] [Acceso el 17 abril de 2019]. Disponible en: http://www.aecc.es/.

2. Registro Nacional de Tumores Infantiles. Cáncer Infantil en España 2017. [Internet] [Acceso el 17 abril de 2019]. Disponible en: http://www.uv.es/rnti/

3. Moreno P. El sentimiento de autoeficacia en pacientes de oncología infantil: desarrollo de un instrumento de medida. [Internet] Tesis doctoral. Universidad Complutense de Madrid; 2015 [Acceso el 17 abril de 2019]. Disponible en: http://eprints.ucm.es/40391/

4. Benight CC, Flores J, Tashiro T. Bereavement coping self-efficacy in cancer widows, Death Stud 2001; 25: 97-125. doi: 10.1080/07481180125921

5. Hagedoorn M, Molleman E. Facial disfigurement in patients with head and neck cancer: the role of social self-efficacy. Health Psychol 2006; 25: 643-7. doi: 10.1037/02786133.25.5.643

6. Liang SY, Yates P, Edwards H, Tsay SL. Factors influencing opioid-taking self-efficacy and analgesic adherence in Taiwanese outpatients with cancer. Psychooncology 2008; 17 : 1100-7. doi: 10.1002/pon.1326

7. Curtis R, Groarke AM, Sullivan F. Stress and self-efficacy predict psychological adjustment at diagnosis of prostate cancer. Sci Rep 2014; 4: 1-5. doi: 10.1038/srep05569 
8. Porter LS, Keefe FJ, Garst J, McBride CM, Baucom, D. Self-efficacy for managing pain, symptoms, and function in patients with lung cancer and their informal caregivers: Associations with symptoms and distress. Pain 2008; 137: 306-15. doi: 10.1016/j. pain.2007.09.010

9. Collie K, Wong P, Tilston J, Butler LD, Turner-Cobb J, Kreshka MA, et al. Self-efficacy, coping, and difficulties interacting with health care professionals among women living with breast cancer in rural communities. Psychooncology 2005; 14: 901-12. doi: 10.1002/ pon.944

10. Bandura A. Guide for constructing self-efficacy scales. En: Pajares F, Urdan T, editors. Adolescence and education. Self-efficacy beliefs of adolescents. Vol. 4. Greenwich, CT: Information Age Pub; 2006. p. 307-37.

11. Morales P. Medición de actitudes en Psicología y Educación: construcción de escalas y problemas metodológicos. $3^{\mathrm{a}} \mathrm{ed}$. rev. Madrid: Universidad Pontificia de Comillas; 2006.

12. Morales P. Guía para construir cuestionarios y escalas de actitudes. [Internet] 2011a [Acceso el 17 abril de 2019]. Disponible en: http://www.upcomillas.es/personal/peter/ otrosdocumentos/Guiaparaconstruirescalasdeactitudes.pdf

13. Morales P. Análisis factorial en la construcción e interpretación de test, escalas y cuestionario. [Internet] 2011b [Acceso el 17 abril de 2019]. Disponible en: http://www. upcomillas.es/personal/peter/investigacion/AnalisisFactorial.pdf

14. Nunnally JC, Bernstein IJ. Teoría psicométrica. México D.F.: McGraw-Hill; 1995.

15. Costello AB, Osborne JW. Best practices in exploratory factor análisis: four recommendations for getting the most from your analysis. Pract Assess Res Eva 2005; 10: 1-9. [Acceso el 17 abril de 2019]. Disponible en: http://pareonline.net/getvn. $\operatorname{asp} ? \mathrm{v}=10 \& \mathrm{n}=7$

16. López-Fernández O, Honrubia-Serrano ML, Freixa-Blanxart M. Adaptación española del "Mobile Phone Problem Use Scale" para población adolescente. Adicciones 2012; 24: 123-30.

17. Bragado C. Funcionamiento psicosocial e intervenciones psicológicas en niños con cáncer. Psicooncología 2009; 6: 327-41.

18. Crespo D, Muñoz A. Aspectos psicosociales del cáncer en la infancia. Rev Esp Pediatr 2013; 69: 248-52.

19. Domínguez M. El cáncer desde la mirada del niño. Madrid: Alianza; 2009.

20. González YR. Depresión en niños y niñas con cáncer. Actual Psicol 2006; 20: 22-44. doi: 10.15517/ap.v20i107.35

21. Méndez X, Orgilés M, López-Roig S, Espada JP. Atención psicológica en el cáncer infantil. Psicooncología 2004; 1: 139-54.

22. Noreña AL, Cibanal L. El contexto de la interacción comunicativa. Factores que influyen en la comunicación entre los profesionales de enfermería y los niños hospitalizados. Cultura de los Cuidados 2008; 23: 70-9. doi: 10.14198/cuid.2008.23.09

23. Cely-Aranda JC, Duque CP, Capafons A. Psicooncología pediátrica. Diversitas 2013; 9: 289-304. doi: 10.15332/s1794-9998.2013.0002.04

24. Die M. El niño y el adolescente con cáncer. En: Die M, editor. Psico-Oncología. Madrid: Ades, 2003.

25. Hedström M, Haglund K, Skolin I, Von Essen L. Distressing events for children and adolescents with cancer: child, parent and nurse perceptions. J Pediatr Oncol Nurs 2003; 20: 120-32. doi: 10.1053/jpon.2003.76 
26. Salas M, Gabaldón O, Mayoral JL, Arce M, Amayra I. Evaluación y control de síntomas en oncología pediátrica: Una necesidad que cubrir y un mundo por descubrir. Psicooncología 2004; 1(2-3): 231-50.

27. Fan S, Eiser C. Body image of children and adolescents with cancer: A systematic review. Body Image 2009; 6: 247-56. doi:10.1016/j.bodyim.2009.06.002

28. Vanegas BC, Beltrán ME, Cifuentes V, Duarte YA, Montoya JJ, Rivera JM, et al. Vivencias psicosociales reveladas por niños que reciben tratamiento con quimioterapia por cáncer. Av Enferm 2009; 27: 102-12.

29. Bernabeu J, Fournier C, García-Cuenca E, Moran M, Plasencia M, Prades O, et al. Atención interdisciplinar a las secuelas de la enfermedad y/o tratamientos en oncología pediátrica. Psicooncología 2009; 6: 381-411.

30. González-Arratia NI, Nieto D, Valdez JL. Resiliencia en madres e hijos con cáncer. Psicooncología 2011; 8: 113-23.

31. Noll RB, Kupst MJ. Commentary: The psychological impact of pediatric cancer hardiness, the exception or the rule? J Pediatr Psychol 2007; 32: 1089-98. doi: 10.1093/jpepsy/ jsm049

32. Canning EH, Canning RD, Boyce WT. Depressive sympthoms and adaptive style in children with cancer. J Am Acad Child Adolesc Psychiatry 1992; 31: 1120-4. doi: 10.1097/00004583-199211000-00021

33. Dejong M, Fombonne E. Depression in paediatric cancer: An overview. Psychooncology 2006; 15: 553-66. doi:10.1002/pon.1002

34. Bragado C, Hernández-Lloreda MJ, Sánchez-Bernardos ML, Urbano S. Autoconcepto físico, ansiedad, depresión y autoestima en niños con cáncer y niños sanos sin historia de cáncer. Psicothema 2008; 20: 413-9.

35. Clarke SA, Eiser C. Health behaviours in childhood cancer survivors: A systematic review. Eur J Cancer 2007; 43: 1373-84. doi: 10.1016/j.ejca.2007.03.002

36. Eiser C, Hill JJ, Vance YH. Examining the psychological consequences of surviving childhood cancer: Systematic review as a research method in pediatric psychology. J Pediatr Psychol 2000; 25: 449-60. doi: 10.1093/jpepsy/25.6.449

37. Grootenhuis MA, Last BF. Children with cancer with different survival perspectives: Defensiveness, control strategies, and psychological adjustment. Psychooncology 2001; 10: 305-14. doi: 10.1002/pon.529

38. Noll RB, Gartstein MA, Vannatta K, Correll J, Bukowski WM, Davies WH. Social, emotional and behavioral functioning of children with cancer. Pediatrics1999; 103: 71-8.

39. Patenaude AF, Kupst MJ. Psychosocial functioning in pediatric cancer. J Pediatr Psychol 2005; 30: 9-27. doi: 10.1093/jpepsy/jsi012 